\title{
The follow-up management of non-metastatic renal cell carcinoma: definition of a surveillance protocol
}

\author{
Alessandro Antonelli, Alberto Cozzoli, Danilo Zani, Tiziano Zanotelli, Maria Nicolai, \\ Sergio Cosciani Cunico and Claudio Simeone \\ Department of Urology, University of Brescia, Brescia, Italy
}

Accepted for publication 18 September 2006

\section{OBJECTIVE}

To define a follow-up protocol based on the University of California Los Angeles Integrated Staging System (UISS) for patients undergoing surgery for NOMO renal cell carcinoma (RCC).

\section{PATIENTS AND METHODS}

The clinical records of patients treated with radical surgery for NO/NXMO RCC and monitored through periodic follow-up studies ( $\geq 24$ months in disease-free patients) were reviewed retrospectively from 1399 patients surgically treated for renal neoplasms between 1983 and 2005. Each case was assigned a UISS risk category; recurrence features, time and site were recorded. In particular, recurrence sites were categorized into local, renal (ipsilateral or contralateral) and distant (single-site or disseminated).

RESULTS

The records were reviewed of 814 patients with a mean follow-up of 75.6 months. UISS risk categories were distributed as follows: high-risk (HR) 17.2\%, intermediate-risk (IR) 51.6\% and low-risk (LR) 31.2\%. Disease-free survival rates at 5 years were $63.9 \%, 88.3 \%$ and $96.5 \%$ (log-rank test $P<0.001)$, respectively. The disease recurred in 193 patients (23.7\%), at distant sites $(73.0 \%$ of recurrences), locally (11.9\%), in the contralateral kidney (10.9\%) and in the ipsilateral kidney (4.1\%). There was a significant correlation between UISS category and risk of distant or local (both $P<0.001$ ) recurrences, whereas there was no correlation of recurrences in the operated kidney $(P=0.372)$ or contralateral kidney $(P=0.898)$.

\section{CONCLUSIONS}

The prognostic accuracy and applicability of the UISS for distant and local recurrences is confirmed, whereas renal relapses have an independent course. A follow-up scheme tailored to the recurrence patterns observed in each UISS risk group is recommended.

\section{KEYWORDS}

renal cell carcinoma, recurrence, follow-up, UCLA integrated staging system

\section{INTRODUCTION}

Of patients undergoing radical nephrectomy for non-metastatic RCC at diagnosis, 20-40\% have disease recurrence during the follow-up [1]. Even though the biological behaviour of renal neoplasms remains unpredictable, the connection between prognosis and several tumour- and host-related risk factors, when considered both individually and combined within integrated staging systems, has been widely confirmed [2]. The University of California Los Angeles Integrated Staging System (UISS) [3], amongst others, stands out for its accuracy, user-friendliness and reproducibility, and for use with external data sets [4].

The purpose of the present retrospective study was to verify the time and patterns of the recurrences recorded in many patients with NO/NXMO RCC who had been treated and monitored at one institution, and thus to recommend a targeted follow-up scheme.

\section{PATIENTS AND METHODS}

Between January 1983 and December 2005, the data from 1399 consecutive patients treated surgically for parenchymal renal neoplasm and monitored through periodic follow-up studies (every 6 months for the first 2 years and then every year) including a physical examination, laboratory tests (kidney and liver function tests, blood tests, serum protein electrophoresis, calcaemia and alkaline phosphatase) and targeted chest (X-ray or CT) and abdomen (ultrasonography or $\mathrm{CT}$ ) examinations were recorded in a dedicated database. Further abdominal CT was scheduled for 4 months after surgery to all patients treated by conservative surgery, to exclude disease persistence.

An anatomical and pathological evaluation was carried out over the follow-up by two uropathologists; the 1997 TNM staging method was used [5] and tumour histological types were reassigned according to
Heidelberg classification [6], with cytonuclear grading defined according to the Fuhrman system [7].

We reviewed retrospectively the records of patients treated radically (with no clinical or histological evidence of disease persistence) for NO/NXMO RCC at diagnosis and monitored through follow-up studies, for $\geq 24$ months in disease-free patients.

By integrating stage, grade and Eastern Cooperative Oncology Group (ECOG) performance status [8], the UISS risk category for non-metastatic carcinoma was assigned as low-, intermediate- or high-risk (LR, IR and HR) [3] (Table 1). Recurrence time and sites were then recorded and categorized according to the following nomenclature: operated kidney; contralateral kidney; local (lumbar fossa, retroperitoneal lymph nodes, neoplastic caval thrombosis); distant (chest, abdomen, superficial, bone, brain or disseminated). 


TABLE 1 The UISS definitions
\begin{tabular}{ll} 
UISS & Stage \\
\hline LR & PT1 G1-2 ECOG 0 \\
IR & PT1 G3-4 or ECOG $>0$ \\
& pT2 \\
PT3 G1 or ECOG 0 \\
HR & pT3 G $>1$ and ECOG $>0$ \\
& pT4
\end{tabular}

The significance of differences between continuous variables was estimated using a t-test, and between nominal variables using the chi-square test; survival was analysed using the Kaplan-Meier method and differences were estimated using the log-rank test. In all tests two-tailed $P$ values were used and regarded as statistically significant if $<0.05$.

\section{RESULTS}

We selected the records of 814 patients (mean age 61.7 years, range $20.6-85.5$; male-tofemale ratio $1.5: 1)$ who had either conservative surgery (158) or nephrectomy (656) for NO/NXMO RCC at diagnosis. The most frequent tumour histological types were conventional (88.9\%), tubulo-papillary (6.1\%) and chromophobic (3.6\%).

The mean (SD, range) overall follow-up was $75.6(48.3,4-296)$ months and 82.9 (47.9) months for currently disease-free patients. There were no significant differences in mean follow-up between UISS risk categories (LR 76.1 months, IR 79.2, HR 68.4). Table 2 shows the UISS stage distribution.

The disease progressed in 193 patients (23.7\% of 814); the cause-specific survival at 5 years was $96.5 \%, 88.3 \%$ and $63.9 \%$ for $L R, I R$ and HR stages, respectively (log-rank test $P<0.001)$. In particular, 53.6\% (75), 21.9\% (92) and 10.2\% (26) patients at $H R, I R$ and $L R$ developed a recurrence in the site and at the time shown in Table 3. The difference in disease progression rates among the three UISS risk categories was statistically significant $(P<0.001)$. There was a significant correlation between UISS stage and risk of distant or local (both $P<0.001$ ) recurrence, whereas there was no correlation with the operated $(P=0.372)$ or contralateral $(P=0.898)$ kidney, with prevailing

\begin{tabular}{|c|c|c|c|c|}
\hline \multirow[b]{2}{*}{ Surgery, n (\%) } & \multicolumn{3}{|l|}{ UISS stage } & \multirow{2}{*}{$\begin{array}{l}\text { TABLE } 2 \\
\text { The UISS stage distribution }\end{array}$} \\
\hline & $\overline{\mathrm{HR}}$ & $\mathrm{IR}$ & $\overline{L R}$ & \\
\hline Conservative & $2(1.3)$ & $70(44.3)$ & $86(54.4)$ & and type of surgery selected \\
\hline Radical nephrectomy & $138(21.0)$ & $350(53.4)$ & $168(25.6)$ & for the 814 patients \\
\hline Total & $140(17.2)$ & $420(51.6)$ & $254(25.2)$ & reviewed \\
\hline
\end{tabular}

TABLE 3 Recurrence sites and time, as a percentage of asymptomatic patients, and the distribution of different types of recurrence in UISS risk groups (percentages in bold are calculated only for patients with distant metastasis, showing the distribution of each site for all distant metastasis).

\begin{tabular}{|c|c|c|c|c|c|c|}
\hline Site & $\begin{array}{l}N(\%) \text { of } \\
\text { patients }\end{array}$ & $\begin{array}{l}\text { Mean time, } \\
\text { months }\end{array}$ & Asymptomatic & $L R$ & IR & $H R$ \\
\hline Operated kidney & $8(4.1)$ & 23.4 & 100.0 & 11.6 & 5.4 & 0 \\
\hline Contralateral kidney & $21(10.9)$ & 71.8 & 95.2 & 30.1 & 10.1 & 4.0 \\
\hline Local recurrence & $23(11.9)$ & 26.4 & 69.5 & 3.9 & 7.6 & 20.0 \\
\hline Distant recurrence & 141 (73.0) & 29.5 & 73.0 & 53.8 & 76.1 & 76.0 \\
\hline Abdomen & $22(15.6)$ & 32.8 & 86.4 & 7.2 & 17.1 & 15.8 \\
\hline Chest & $68(48.3)$ & 29.5 & 86.8 & 35.7 & 50.0 & 49.1 \\
\hline Bone & $16(11.3)$ & 14.9 & 31.2 & 21.4 & 12.9 & 7.0 \\
\hline Brain & $5(3.5)$ & 16.9 & 0 & 0 & 2.9 & 5.3 \\
\hline Superficial & $9(6.4)$ & 55.1 & 81.0 & 21.4 & 5.7 & 3.5 \\
\hline Diffused & $21(14.9)$ & 24.1 & 52.4 & 14.3 & 11.4 & 19.3 \\
\hline
\end{tabular}

proportions in lower risk categories. UISS staging correlated with mean time to recurrence, although it was statistically insignificant (LR 53.5 months, IR 36.1, HR 30.0). Figure 1a-c shows the recurrence distribution of isolated thoracic, abdominal (abdominal metastases, renal recurrences, local recurrences) and bone recurrences over time in each risk group.

There was a significantly lower risk of recurrence in general in patients treated with nephron-sparing surgery than nephrectomy (5.6\% vs $19.5 \%, P<0.001$ ). Nevertheless, when data were stratified based on UISS stage there were no significant differences (LR $P=0.430 ; \mathrm{IR} P=0.170 ; \mathrm{HR} P=0.919$ ). Likewise, the mean time to recurrence, at 36.7 and 36.0 months, respectively, was similar for the two groups.

In 95.3\% of patients who had recurrent disease there was a conventional RCC; in particular, recurrences affected 25.4\%, 12.0\% and no patients with conventional, tubulopapillary and chromophobic RCC, respectively. Data stratification based on UISS stage showed no statistically significant differences in progression rates between conventional and papillary RCC (LR $P=0.448$; IR $P=0.141$; $\mathrm{HR} P=0.513)$.
Of 193 recurrences, 133 were treated with curative intent; in particular, 93 patients were treated with surgery, 31 with chemoimmunotherapy and nine with radiotherapy. Disease recurrence was detected at a still asymptomatic stage in 147 patients (76.2\%); 50 showed no further signs of disease within a mean follow-up of 53.8 months after recurrence, 48 of them had reported no symptoms at diagnosis, while 49 of them had been treated with surgery. In general, patients whose recurrence was treated had a higher survival rate than had untreated patients (survival at 24 months after recurrence was $74.7 \%$ vs $14.2 \%, P<0.001)$. Patients with asymptomatic recurrences had a significantly higher survival rate from disease recurrence (survival at 24 months after recurrence was $62.6 \%$ vs $29.5 \%, P<0.001)$. Finally, patients with an isolated recurrence had better survival rates than those with multiple-site recurrences (survival at 24 months after recurrence was $62.8 \%$ vs $24.8 \%, P<0.001$ ).

\section{DISCUSSION}

Despite the dramatic increase in the rate of early diagnoses in last two decades, RCC remains the urological malignancy associated with the highest mortality rate [9]. This is 
explained by the lack of effective systemic treatments for advanced disease, which is detected at diagnosis in 20-30\% of patients or during the follow-up in $20-40 \%$ of those with organ-confined disease at diagnosis [1].

After initial recommendations for intensive, indiscriminate follow-up [10], tailored surveillance protocols based on TNM staging were defined, with minimal requirements for low but also for more advanced stages, considering the lack of successful treatments for recurrences [11-13]. More recently, the development of staging systems integrating several individually acknowledged prognostic factors has led to the definition of groups of patients with a better diversified recurrence risk [14-22] and to the creation of different follow-up protocols. Following the review of 559 patients with non-metastatic disease (but $40 \mathrm{~N}+$ cases), stratified according to UISS stage, system developers recently recommended the first surveillance scheme based on clinical evidence [23].

The UISS integrates clinical factors commonly recorded (stage, grade and ECOG score) and so, besides having a tried and tested prognostic value, it can be easily applied also to external data sets, as was shown recently $[4,24]$. Moreover, comprehensive case reviews [19] showed the treatment-independent prognostic role played by several host-related factors in metastatic patients (performance status, weight loss, lactate dehydrogenase, haemoglobin level, calcium, sedimentation rate, etc.), and the use of a performance indicator (ECOG score) to stratify also nonmetastatic cases certainly supports the validity of the UISS, confirming the prognostic role of symptoms at diagnosis.

Likewise, in the present study, the UISS was used for 814 consecutive patients treated with radical surgery for non-metastatic RCC (NO/NXMO) at a University centre other than the centre where the system was designed. All patients were monitored through an intensive, indiscriminate and long-term $(\geq 24$ months in disease-free patients, 80 months on average) follow-up protocol.

The data presented confirm that radical surgery for non-metastatic RCC is associated with a considerable rate of recurrence (23.7\%), which mainly takes the form of distant metastases (73\% of recurrences). The high proportion of asymptomatic and isolated recurrences would seem to indicate the good sensitivity of the surveillance approach applied. However, the large majority of patients who never had progression were excessively monitored, causing a concomitant increase in costs.

Also in our experience, the use of the UISS led to the appropriate prognostic discrimination of patients, as shown by the statistically significant differences resulting from the comparison between recurrence and diseasefree survival rates. Therefore, we confirm that the system is fully applicable to external data sets, with recurrence rates comparable with those reported by Lam et al. [23], even though in our experience the survival rates were generally higher and differences between LR and IR patients significant but not so marked.

There was a substantial correlation between UISS category and the risk of local and distant recurrences, and with time to recurrence, which was shorter for the HR and IR groups. Conversely, there was no correlation between UISS stage and risk of renal recurrences, which were more frequent in the LR and IR groups. The rare event of recurrences in kidneys treated with conservative surgery was always in the first years of follow-up and should be attributed to underestimated multifocality or to technical and surgical factors, which are less related to the factors considered by the staging system. As to isolated recurrences in the contralateral kidney, the present long mean latency (71.8 months, range 7-291) suggests the potential development of a second independent neoplasm, which should not be considered a true 'disease recurrence'.

However, renal recurrences might also be related to previous or contemporary metastatic disease at other sites (17 cases in the present study, mean latency 41 months, range 8-121), which would otherwise indicate the potential metastatic nature of the second renal neoplasm. Probably only by comparing the tumour genome of the two neoplasms could these two conditions be differentiated to direct the therapy (systemic vs local) and follow-up.

Unlike other validated prognostic systems, e.g. that suggested by Kattan et al. [16], the UISS does not rely on the tumour histological type. In our experience, chromophobic RCCs had an extremely favourable prognosis and we think that they should be managed as for LR cases, with the sole aim of preventing the effects of an incorrect histological diagnosis.
FIG. 1. The time distribution (marks representing the events of recurrence as percentiles on overall recurrences in each specific site) of: $a$, chest; $b$, abdominal; and c, bone recurrences.

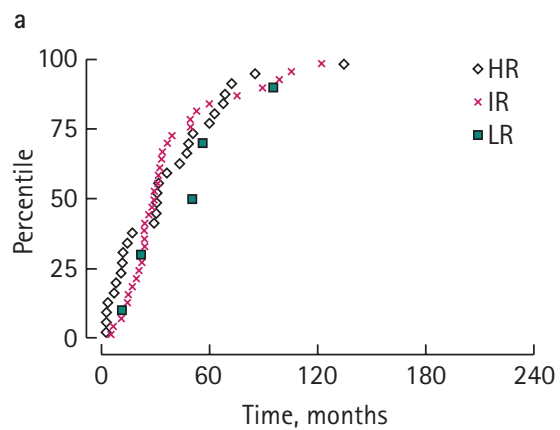

b

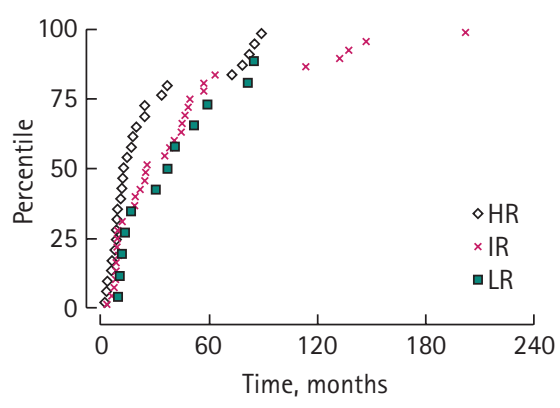

c

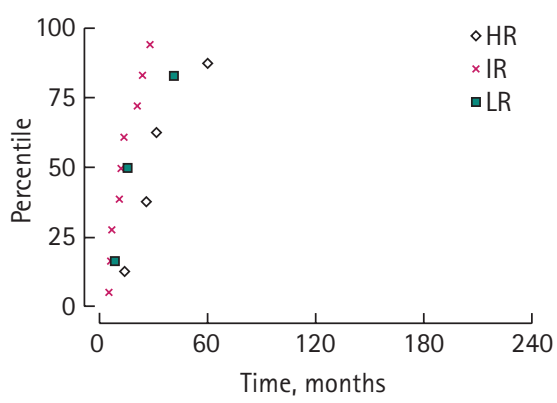

Conversely, the differences between conventional and papillary RCC were not significant once the UISS was applied, and we think that this distinction should not be included.

Recurrence time and site distribution (Fig. 1 and Table 3) show that the LR group had a low risk of abdominal recurrence, with a prevalence of renal recurrence, and a very low risk of thoracic metastasis. By contrast, the IR and $H R$ groups had a risk, respectively, that was higher for recurrence both in the abdomen, usually with distant or local relapses, or in the chest. In the following 5 years for $L R$ patients there was a further 
TABLE 4 The follow-up scheme based on UISS risk categories

\begin{tabular}{llll} 
Group & Years of follow-up & & \\
\cline { 2 - 4 } & $0-5$ & $6-10$ & $>10$ \\
\hline LR & $\begin{array}{c}\text { Thoracic study every } 30 \text { months } \\
\text { and abdominal study yearly }\end{array}$ & $\begin{array}{c}\text { Thoracic and abdominal } \\
\text { study every } 30 \text { months }\end{array}$ & $\begin{array}{c}\text { Abdominal study every } \\
5 \text { years }\end{array}$ \\
IR & $\begin{array}{c}\text { Thoracic and abdominal } \\
\text { study every } 6 \text { months }\end{array}$ & $\begin{array}{c}\text { Thoracic study yearly and } \\
\text { abdominal study every }\end{array}$ & $\begin{array}{c}\text { Abdominal study every } \\
5 \text { years }\end{array}$ \\
& & 30 months & \\
HR & Thoracic and abdominal study & Thoracic and abdominal & Abdominal study every \\
& every 6 months & study yearly & 5 years
\end{tabular}

reduction in the risk, whereas fewer but significantly many recurrences, mainly in the chest and less in the abdomen, persisted for the IR and HR patients. After 10 years of follow-up recurrences were extremely rare and almost exclusively in the contralateral kidney. The follow-up scheme suggested (Table 4), which is based on these results, resembles the recommendations for the UISS [24]. We think that the additional follow-up after nephron-sparing surgery is unnecessary because the risk and time of recurrence is statistically equal to nephrectomy when the UISS is applied. Moreover, the rare event of a relapse in the operated kidney is prevented by the indication for abdominal studies in the first 5 years for all the UISS categories.

Because the present study was retrospective it is difficult to compare the risk-benefit ratios for each diagnostic method (X-ray, ultrasonography, CT, etc.) as a first-line survey. From our experience, we think it is reasonable to use more sensitive methods (thoracicabdominal CT) in the first 5 years of followup, extensively for HR and less for IR patients. For LR patients, and after 5 years also for HR and IR patients, a chest $X$-ray and abdominal ultrasonography could direct second-line tests, their low cost and minimal invasiveness being proportionate to the reduced risk of recurrence.

Many studies confirm that a diagnosis of bone metastasis can be predicted from its symptoms, alkaline phosphatase values or the presence of extra bone metastases, making routine bone scintigraphy questionable, especially for LR patients and when the ECOG score is 0 [23]. Bone scanning was not a routine examination in our follow-up programme. Therefore, the diagnoses of isolated bone metastasis generally followed the appearance of symptoms or alterations in alkaline phosphatase levels, and they were rarely diagnosed when asymptomatic from bone anomalies on the chest or abdominal studies. Isolated bone metastases were detected in all three UISS categories (Fig. 1c) and therefore it can be supposed that a more extensive use of bone scintigraphy could help to estimate the real incidence of metastasis at this site and possibly anticipate the diagnosis. However, the fatal prognosis and the smallness of this group of patients make a consensus for this indication unlikely, and probably only a prospective evaluation could critically establish the real need for bone scanning.

Cerebral lesions were rare and developed only in IR or HR patients. For these categories the need for chest-abdominal CT could justify its occasional extension to the brain with no excessive increase in costs. Physical examinations and interviews with patients, together with serum and chemistry tests, should be included in long-term annual evaluation for all cases.

As an extensive recent review of previous reports [25] showed better results for survival when recurrences were asymptomatic, resectable and isolated, confirming that, given the lack of effective systemic therapies, early diagnosis can make the relapse salvageable and justify a careful follow-up for patients with RCC.

In conclusion, non-metastatic RCCs recur in $\approx 25 \%$ of cases, mainly as distant metastases, even after being treated radically. The results obtained with treatable recurrences and the high proportion of asymptomatic diagnoses support the need for a long-term follow-up, even though the lack of efficient systemic therapies means that prospects for treating patients with recurrence are rather poor.
We confirm the prognostic accuracy and repeatability of the UISS for local and distant recurrences, whereas renal relapses were independent of this system.

\section{CONFLICT OF INTEREST}

None declared.

\section{REFERENCES}

1 Janzen NK, Kim HL, Figlin RA, Belldegrun AS. Surveillance after radical or partial nephrectomy for localized renal cell carcinoma and management of recurrent disease. Urol Clin North Am 2003; 30: 843-52

2 Elson PJ, Witte RS, Trump DL. Prognostic factors for survival in patients with recurrent or metastatic renal cell carcinoma. Cancer Res 1988; 48: 7310-3

3 Zisman A, Pantuck AJ, Dorey F et al. Improved prognostication of renal cell carcinoma using an integrated staging system. J Clin Oncol 2001; 19: 1649-57

4 Han KR, Bleumer I, Pantuck AJ et al. Validation of an integrated staging system toward improved prognostication of patients with localized renal cell carcinoma in an international population. J Urol 2003; 170: 2221-4

5 Guinan P, Sobin LH, Algaba F et al. TNM staging of renal cell carcinoma: Workgroup no. 3. Union International Contre le Cancer (UICC) and the American Joint Committee on Cancer (AJCC). Cancer 1997; 80: 992-3

6 Kovacs G, Akhtar M, Beckwith BJ et al. The Heidelberg classification of renal cell tumours. J Pathol 1997; 183: 131-3

7 Fuhrman SA, Lasky LC, Limas C. Prognostic significance of morphologic parameters in renal cell carcinoma. Am J Surg Pathol 1982; 6: 655-63

8 Oken MM, Creech RH, Tormey DC et al. Toxicity and response criteria of the Eastern Cooperative Oncology Group. Am J Clin Oncol 1982; 5: 649-55

9 Jemal A, Tiwari RC, Murray T et al. Cancer statistics 2004. CA Cancer J Clin 2004; 54: 8-29

10 Montie JE. Follow-up after partial or total nephrectomy for renal cell carcinoma. Urol Clin North Am 1994; 21 : 589-92

11 Sandock DS, Seftel AD, Resnick MI. A new protocol for the follow-up of renal 
cell carcinoma based on pathological stage. J Urol 1995; 154: 28-31

12 Levy DA, Slaton JW, Swanson DA, Dinney CP. Stage specific guidelines for surveillance after radical nephrectomy for local renal cell carcinoma. J Urol 1998; 159: 1163-7

13 Hafez KS, Novick AC, Campbell SC. Patterns of tumour recurrence and guidelines for follow-up after nephron sparing surgery for sporadic renal cell carcinoma. J Urol 1997; 157: 2067-70

14 Abou-Jawde RM, Mekhail T, Bou Merhi GF. Prognostic factors (PF) for survival in previously untreated metastatic renal cell cancer (RCC). A comprehensive evaluation and validation of established risk groups. Proc Am Soc Clin Oncol 2003; 22: 385, Abstract 1545

15 Frank I, Blute ML, Cheville JC, Lohse CM, Weaver L, Zincke H. An outcome prediction model for patients with clear cell renal cell carcinoma treated with radical nephrectomy based on tumour stage, size, grade and necrosis: the SSIGN score. J Urol 2002; 168: 2395400

16 Kattan MW, Reuter V, Motzer RJ, Katz J, Russo P. A postoperative prognostic nomogram for renal cell carcinoma. J Urol 2001; 166: 63-7
17 Lam JS, Shvarts 0, Leppert JT, Figlin RA, Belldegrun AS. Renal cell carcinoma 2005: new frontiers in staging, prognostication and targeted molecular therapy. J Urol 2005; 173: 1853-62

18 Ljungberg B, Alamdari FI, Rasmuson T, Roos G. Follow-up guidelines for nonmetastatic renal cell carcinoma based on the occurrence of metastases after radical nephrectomy. BJU Int 1999; 84: 405-11

19 Motzer RJ, Mazumdar M, Bacik J, Berg W, Amsterdam A, Ferrara J. Survival and prognostic stratification of 670 patients with advanced renal cell carcinoma. J Clin Oncol 1999; 17: 2530-40

20 Stephenson AJ, Chetner MP, Rourke K et al. Guidelines for the surveillance of localized renal cell carcinoma based on the patterns of relapse after nephrectomy. J Urol 2004; 172: 58-62

21 Uzzo R, Novick AC. Surveillance strategies following surgery for renal cell carcinoma. In Figlin R ed., Renal and Adrenal Tumors - Biology and Management. New York: Oxford University Press, 2003: 324-30

22 Zisman A, Pantuck AJ, Wieder J et al. Risk group assessment and clinical outcome algorithm to predict the natural history of patients with surgically resected renal cell carcinoma. J Clin Oncol 2002; 20: 4559-66

23 Lam JS, Shvarts 0, Leppert JT, Pantuck AJ, Figlin RA, Belldegrun AS. Postoperative surveillance protocol for patients with localized and locally advanced renal cell carcinoma based on a validated prognostic nomogram and risk group stratification system. J Urol 2005; 174: 466-72

24 Patard JJ, Kim HL, Lam JS et al. Use of the University of California Los Angeles integrated staging system to predict survival in renal cell carcinoma: an international multicenter study. J Clin Oncol 2004; 22: 3316-22

25 Kuczyk MA, Anastasiadis AG, Zimmermann R, Merseburger AS, Corvin S, Stenzl A. Current aspects of the surgical management of organconfined, metastatic and recurrent renal cell cancer. BJU Int 2005; 96: 721-7

Correspondence: Alessandro Antonelli, Department of Urology, University of Brescia, Brescia, Italy.

e-mail: alxanto@hotmail.com

Abbreviations: UISS, University of California Los Angeles Integrated Staging System; ECOG, Eastern Cooperative Oncology Group; $L R, I R, H R$, low-, intermediate-, or high-risk. 